\section{Fièvre hémorragique à virus Ebola: un vaccin en dose unique efficace chez le primate}

Sylvain Baize, Vincent Deubel
Unité de biologie des infec-

tions virales émergentes,

Institut Pasteur,

21, avenue Tony Garnier, 69365 Lyon Cedex 07, France.

vdeubel@cervi-

lyon.inserm.fr

protéines recombinantes n'étaient pas non plus protecteurs. Le développement d'un vaccin contre la FHVE s'est de plus heurté à de nombreuses difficultés, comme la nécessité d'utiliser un laboratoire de niveau P4, mais aussi l'absence de modèle rongeur reproduisant fidèlement les caractéristiques physiopathologiques et immunitaires associées à l'infection humaine.

Des travaux récents ont montré que le contrôle de l'infection par le virus Ebola était lié à l'induction d'une réponse immune à médiation humorale, mais aussi cellulaire (cytotoxique). On a ainsi observé une corrélation entre la survie des patients infectés et l'apparition précoce d'anticorps et de lymphocytes cytotoxiques [5], mais le seul transfert passif d'anticorps ne permet pas de protéger l'animal d'une infection létale [6]. Le vaccin, pour être efficace, doit donc induire une réponse immune à médiation humorale mais également une réponse lymphocytaire cytotoxique. Une approche récemment envisagée a été l'utilisation de vaccins à ADN, dont on sait qu'ils induisent de fortes réponses cellulaires. L'injection à des cobayes d'un plasmide codant pour la glycoprotéine (GP) ou la nucléoprotéine (NP) virale [7] protège les animaux contre une infection létale par le virus Ebola. De même, l'équipe améri-
Figure 1. Historique des épidémies de FHVE en Afrique Centrale. Les différentes épidémies de FHVE (sous-types Zaïre et Soudan) survenues en Afrique Centrale sont figurées, ainsi que la zone où la circulation du virus $\varepsilon b o l a ~ a$ été démontrée chez les grands singes (ovale hachuré rouge).
> Le virus
ARN m
apparter
Ce virus
princi
où il ind
telle
sous-
$(\rightarrow) \mathrm{m} / \mathrm{s}$ p. 1168 $n^{\circ} 1$, p. 13

Tchibanga Pointe-Noire Kinshasa CONGO (DRC) 1995 
caine de N.J. Sullivan et al. associant le Vaccine Research Center du NIH (Bethesda, Maryland, USA) et le Center for Disease Control d'Atlanta (USA), a réussi à protéger des macaques d'une FHVE par une vaccination associant un plasmide exprimant la GP ou la NP et un adénovirus recombinant (porteur d'une délétion le rendant inapte à la réplication) exprimant la GP du virus Ebola [8] $(\rightarrow)$. Cette démarche prometteuse a

$(\rightarrow) \mathrm{m} / \mathrm{s}$

$2001, n^{\circ} 2$, p. 269 cependant fait l'objet de nombreuses critiques. En effet, ce protocole vaccinal était très complexe, nécessitant 4 injections successives et plusieurs mois pour être efficace, un délai qui le rendait inapplicable dans un cadre épidémique. En outre, la dose de virus Ebola utilisée pour tester l'efficacité de la protection des animaux était extrêmement faible (6 unités formant plages, UFP), faisant douter de l'efficacité d'un tel vaccin dans les conditions naturelles d'infection. Les mêmes auteurs, associés cette fois au laboratoire P4 de I'USAMRIID (Fort Detrick, Maryland, USA), ont donc amélioré cette approche et récemment proposé un protocole de vaccination accélérée efficace chez le macaque [9]. Cette approche utilise cette fois une injection unique d'un mélange d'adénovirus exprimant la GP et la NP du virus Ebola. Cette immunisation permet l'induction rapide d'anticorps, dont la concentration est cependant inférieure à celle obtenue dans l'étude précédente, ainsi qu'une réponse lymphocytaire $\operatorname{CD}^{+}$spécifique. Quatre semaines après l'immunisation, les macaques infectés avec une faible (13UFP) ou une forte (1500 UFP) dose de virus Ebola ont survécu à l'infection. La protection semble totale, puisqu'aucune virémie n'a été observée chez les animaux immunisés. L'analyse de la réponse immune au cours de l'infection a montré que la protection était associée à la réponse humorale mais aussi à des réponses lymphocytaires $\mathrm{CD}^{+}$et $\mathrm{CD}^{+}$ spécifiques. Cette approche, qui reste néanmoins à valider chez l'homme, est très intéressante. Comme le suggèrent les auteurs, l'injection d'une dose unique de vaccin permettrait, à l'instar de la variole, de contrôler rapidement les épidémies de FHVE par la vaccination des personnes vivant dans la zone touchée et du personnel médical en charge des patients. De même, les auteurs suggèrent que ce vaccin pourrait être administré aux grands singes afin de limiter la circulation du virus Ebola dans la région. Cependant, cette approche semble pour le moins difficile à mettre en œuvre dans le contexte particulier de la forêt équatoriale. Une autre réserve provient de l'utilisation d'un vecteur viral communément rencontré par I'homme dans les conditions naturelles, avec le risque non négligeable qu'une immunité préalable anti-adénovirus ne diminue l'efficacité de ce vaccin. On peut également regretter que les auteurs n'envisagent pas, à ce jour, une vaccination préventive pour les populations vivant en zone d'endémie, limitées à quelques millions de personnes, ce qui permettrait probablement de restreindre considérablement la survenue d'épidémies humaines.

Bien qu'un long chemin reste à parcourir avant une éventuelle utilisation chez l'homme, le candidat vaccin de N.J. Sullivan et al. représente une approche prometteuse pour le contrôle des FHVE. Enfin, comme le suggèrent les auteurs, cette approche pourrait être appliquée à d'autres fièvres hémorragiques telles que la fièvre de Marburg ou de Lassa. Accelerated vaccination for Ebola hemorrhagic fever

\section{RÉFÉRENCES}

1. Feldmann $\mathrm{H}$, Jones $\mathrm{S}$, Klenk HD, Schnittler HJ. Ebola virus : from discovery to vaccine. Nat Rev Immunol 2003; 3 : 677-85.

2. Walsh PD, Abernethy KA, Bermejo M, et al. Catastrophic ape decline in western equatorial Africa. Nature 2003; 422: 611-4.

3. Georges AJ, Leroy EM, Renaut $A A$, et al. Ebola hemorrhagic fever outbreaks in Gabon, 1994-1997: epidemiologic and health control issues.J Infect Dis 1999; 179 (suppl 1): S65-75.
4. Lupton HW, Lambert RD, Bumgardner DL, Moe JB, Eddy GA. Inactivated vaccine for Ebola virus efficacious in guinea pig model. Lancet 1980; 2: 1294-5.

5. Baize S, Leroy EM, GeorgesCourbot MC, Capron M, Lansoud-Soukate J, Georges AJ. Réponse immune précoce et le contrôle de l'infection par le virus Ebola. Med Sci (Paris) 1999; 15: 1168-72.
6. Jahrling PB, Geisbert J, Swearengen JR, et al. Passive immunization of Ebola virus-infected cynomolgus monkeys with immunoglobulin from hyperimmune horses. Arch Virol 1996; 11 (suppl): 135-40.

7. Xu L, Sanchez A, Yang ZY, et al. Immunization for Ebola virus infection. Nat Med 1998; 4 : 37-42.
8. Sullivan NJ, Sanchez A, Rollin $P \varepsilon$, Yang ZY, Nabel GJ. Development of a preventive vaccine for Ebola virus infection in primates. Nature 2000; 408: 605-9.

9. Sullivan NJ, Geisbert TW, Geisbert JB, et al. Accelerated vaccination for Ebola virus haemorrhagic fever in non-human primates. Nature 2003; 424: 681-4. 